\title{
Orbital squamous cell carcinoma after retinal detachment surgery
}

\author{
K U Löffler, H Witschel
}

\begin{abstract}
We report on a 76-year-old patient with a squamous cell carcinoma of the left orbit. The tumour had no connection with the conjunctiva but was located at the site of an encircling band which had been inserted 13 years before. A major part of the tumour presented as a well circumscribed solid mass within the extraocular tissues next to the inferior equator, but the exenteration specimen also showed tumour extension within the adjacent choroid. Histological examination showed a well differentiated keratinising squamous cell carcinoma with numerous mitotic figures and many epithelial pearls. A thorough examination in search of a primary carcinoma of the lacrimal gland or the sinus, with invasion into the orbit, or an epithelial neoplasm elsewhere suggestive of metastatic disease into the choroid did not reveal any specific pathological findings. Thus the most probable origin of the tumour seems to be epithelium which had been misplaced during retinal detachment surgery and had subsequently undergone malignant transformation.
\end{abstract}

Among orbital tumours the incidence of squamous cell carcinoma is rather low. It presents mainly as metastases, ${ }^{1}$ as an ingrowing sinus or lacrimal sac tumour, ${ }^{2}$ and in rare cases as carcinoma of the lacrimal gland ${ }^{2}$ or as malignant transformation of a dermoid cyst. ${ }^{3}$ Squamous carcinoma of the choroid is likewise uncommon and only known as metastatic disease. ${ }^{45} \mathrm{We}$ present here the clinical and histological features of a patient with co-existing choroidal and orbital squamous cell carcinoma after retinal detachment surgery many years previously and discuss the possible aetiology in this particular case.

\section{Case report}

In September 1975, a 63-year-old non-myopic man presented with bilateral retinal detachment. Both eyes were treated with an encircling band, and visual acuity improved to preoperative values. Follow-up examinations during the next 10 years did not reveal any further fundus abnormalities; both retinae stayed attached.

By 1985 the right eye had developed an Augenklinik, Freiburg, Germany K U Löffler H Witschel

Correspondence to: Dr K U Löffler, Klinikum der Albert-Ludwigs-Universität, Universitäts-Augenklinik, Killianstrasse 5, D-7800 Freiburg, Germany.

Accepted for publication 7 February 1991 being inconspicuous and the posterior segment unchanged. In April 1988 the patient presented with pain and a reduced VA of 2/60 in his left eye. He had suffered from a subretinal haemorrhage in the meantime, which was thought to be

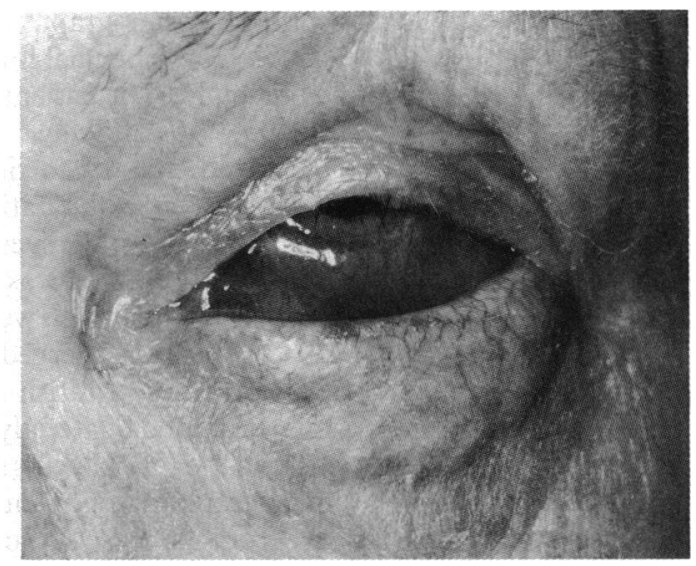

Figure 1 Clinical preoperative photograph of the patient's left eye, presenting with pain, marked episcleral inflammation in the lower quadrants, upward deviation, and conspicuously restricted movements.

responsible for the reduced vision. The intraocular pressure was normal, and there was no evidence of string syndrome such as iris neovascularisation. Artificial tears were prescribed, but a migrainous headache persisted.

Eventually, in November 1988, the VA in the left eye had decreased to hand movements, and there was episcleral inflammation in the lower quadrants with upward deviation of the left globe (Fig 1) and conspicuously restricted movement. Now a retinal detachment and a choroidal mass of unknown origin were noted in the peripheral fundus of the left eye near 6 o'clock, whereas the intraocular anterior segment still appeared normal. On ultrasound the posterior wall of the globe was irregularly thickened. A CT scan showed marked calcification at the site of the encircling band, and a nuclear magnetic resonance scan revealed a circumscribed orbital lesion, $10 \times 5 \mathrm{~mm}$, of possibly granulomatous origin between the encircling band and the inferior rectus muscle. At that time a thorough medical examination as well as several blood tests showed no abnormal findings apart from slight hypertension, diabetes mellitus, and zoster of the

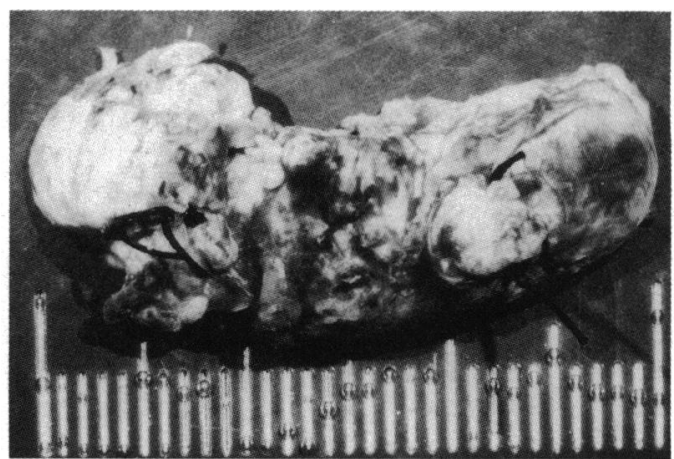

Figure 2 Microscopic appearance of the removed orbital tumour. 


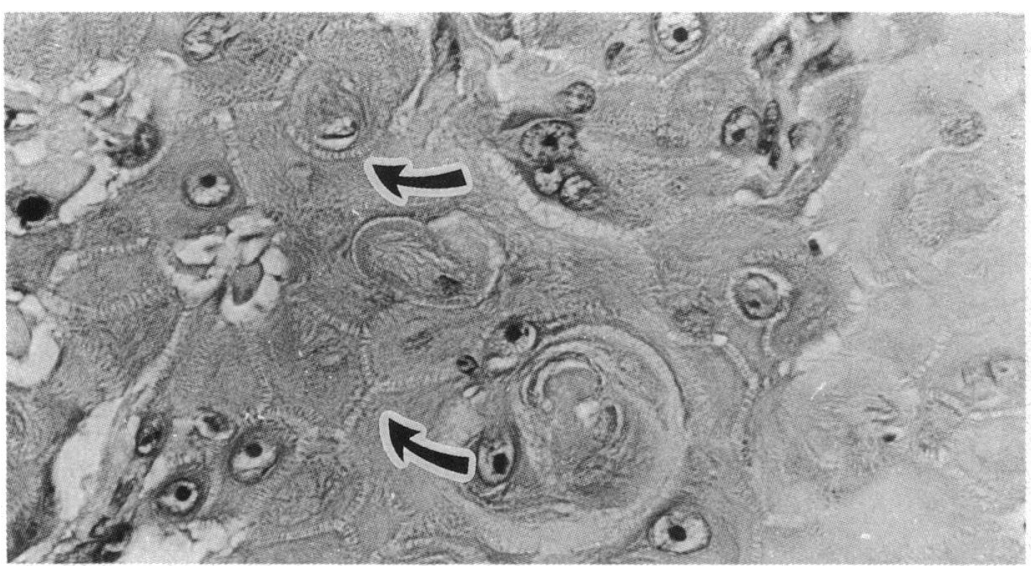

Figure $3 A$

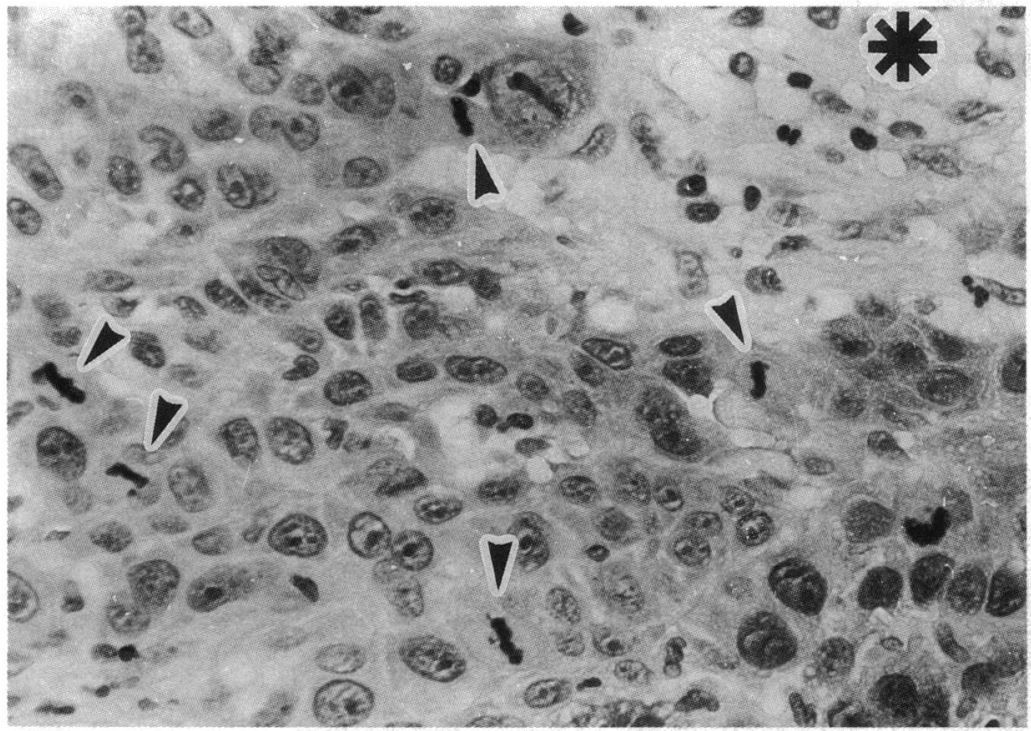

Figure $3 B$

Figure 3 Light microscopic appearance of removed orbital tumour. A: Tumour cells at higher magnification, note numerous prickles (arrows). (Haematoxylin-eosin, $\times 475$.) B: Area with undifferentiated cells, several mitotic figures (arrowheads), and inflammatory infiltrates (star). $(H-E, \times 475$. $)$

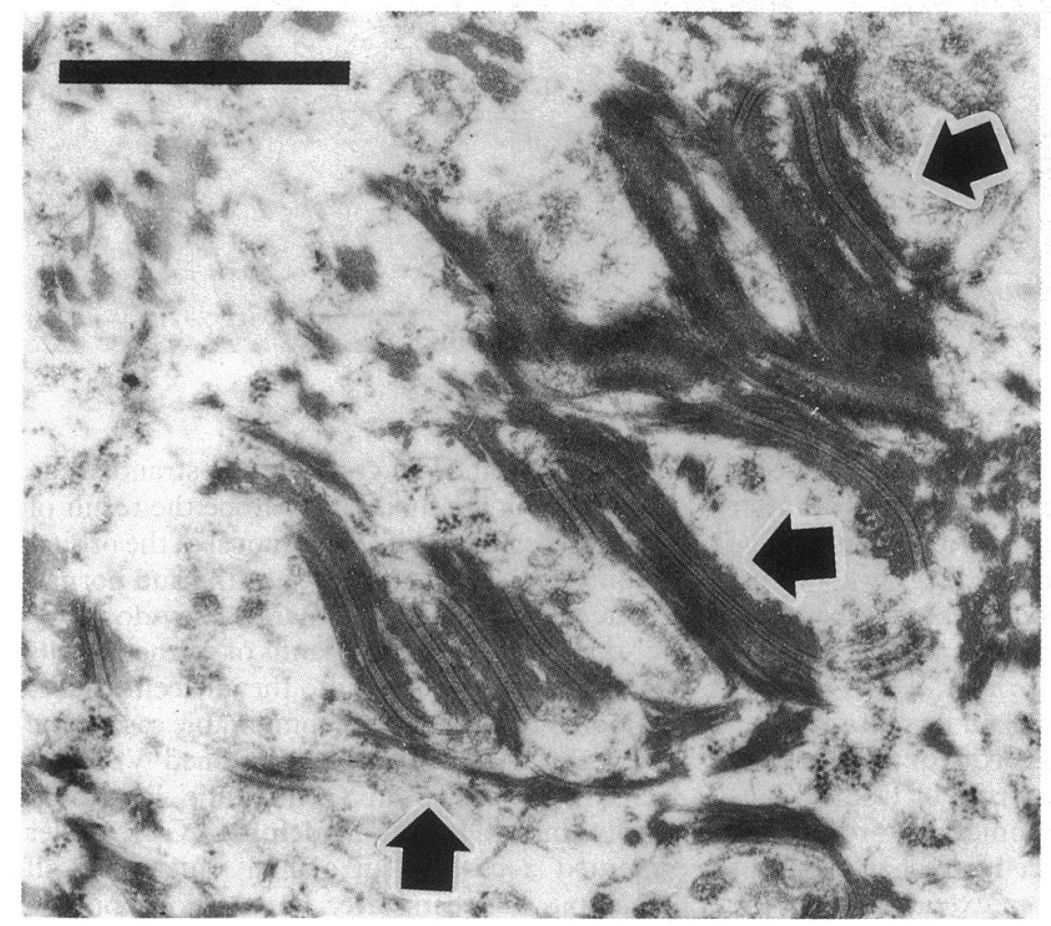

Figure 4 Electron microscope appearance of removed orbital tumour. Note numerous desmosomes (arrows). (Bar=1 $\mu \mathrm{m}$.) trigeminal nerve. One month later, in December 1988, the encircling band was removed and the tumour seen on nuclear magnetic resonance scan was excised. After histological examination of the excised specimen an exenteration was performed in January 1989.

The patient was last seen in June 1990, when he was well and showed no evidence of tumour recurrence, a primary neoplasm elsewhere, or metastatic disease.

\section{HISTOLOGICAL FINDINGS}

The excised tumour measured $19.5 \times 23.5 \times 12$ mm (Fig 2). It was of firm consistency and a whitish colour. Light microscopic examination revealed a keratinising lesion with numerous mitotic figures which was composed mainly of squamous epithelial cells, some of them with definite prickles (Fig 3A). At the periphery the tumour cells seemed to be less differentiated (Fig 3B), and in some parts tumour cells reached the surgical excision line. The connective tissue septa were infiltrated by inflammatory cells. Most tumour cells were arranged in whorls, and nuclear pleomorphism was another prominent feature. Thus the diagnosis of a well differentiated squamous cell carcinoma was made. Electron microscopic investigation confirmed the light microscopic findings, showing typical features of squamous epithelial cells. The tumour cells had irregular nuclei and abundant cytoplasmic tonofibrils with numerous desmosomes (Fig 4) as well as occasional basement membrane formation.

The exenteration specimen was bisected in a vertical plane (Fig 5). Near the superior equator the cavity of the encircling band could be identified, whereas near the inferior equator yellowish tissue had replaced part of the sclera and seemed to join with a pale choroidal tumour $(8 \times 3 \times 8$ mm) (Fig 5). Microscopically this tumour had similar histological features to those of the previously excised orbital lesion, that is, whorls of keratinising squamous cells with interspersed inflammatory cells (Fig 6). However, more areas of necrosis and more prominent infiltration by lymphocytes and plasma cells were conspicuous. Outside the globe only scar tissue with some granulomatous inflammation and muscle giant cells could be found, but there was no evidence of further tumour tissue. The retina was mostly detached and showed early proliferative vitreoretinopathy. In the area of the encircling band mainly above - scleral necrosis with marked calcification (Fig 7) corresponding to the findings by CT scan was present. There was also some chronic conjunctivitis, and some strands of normal conjunctival epithelium extended backwards into the inferior orbit towards the site of the previously excised tumour. Otherwise no significant pathological findings could be identified.

Step sections from the exenteration specimen on the nasal and temporal side including the lacrimal gland were free from tumour.

\section{Discussion}

We present here the clinical and histological 


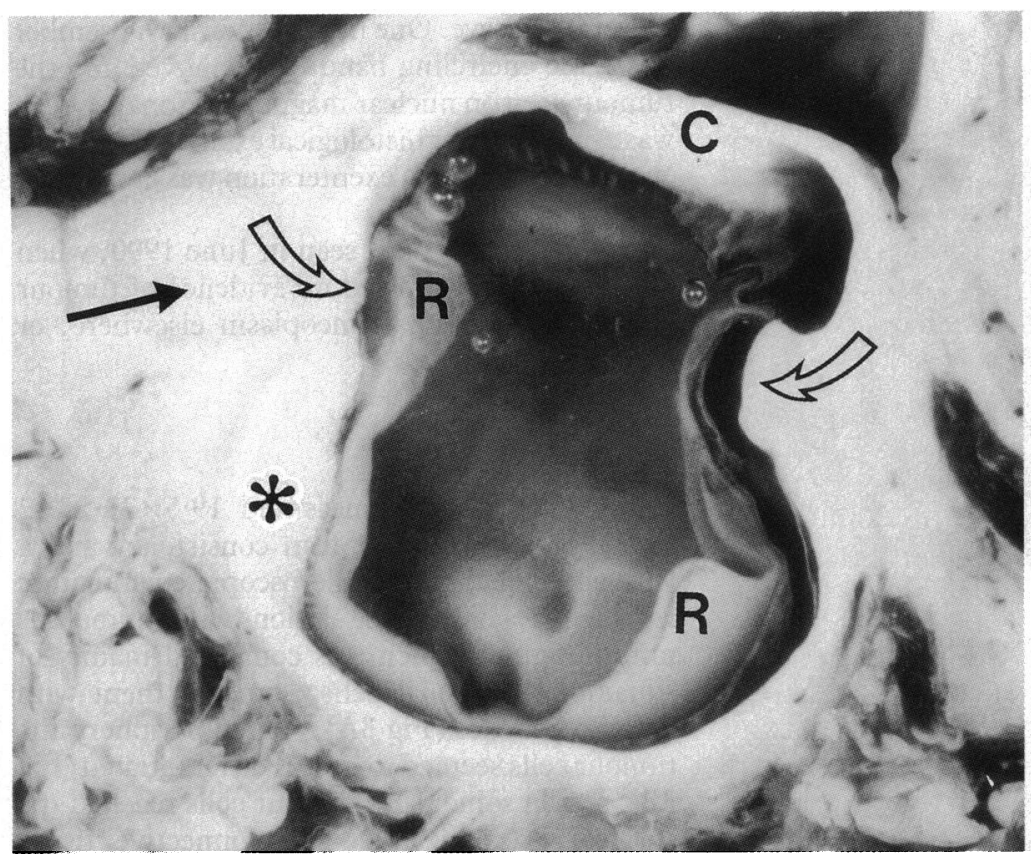

Figure 5 Macroscopic appearance of exenteration specimen $(C=$ cornea. $R=$ retina. $S$ tar $=$ choroidal tumour. Black arrow $=$ site of previous orbital surgery. White arrows $=$ site of removed encircling band). examination, repeated CT scans, and step sections of the exenteration specimen.

Secondly, the possibility of metastatic disease to the choroid from a primary epithelial neoplasm elsewhere with secondary orbital spread has to be investigated. There was, however, no evidence of a primary epithelial neoplasm elsewhere, and in addition the clinical history of pain as a prominent feature, the high degree of differentiation, and the fact that there was more necrosis and reactive inflammation in the choroid than in the orbit favour a primary orbital carcinoma invading the globe. Apart from the apparent absence of a primary tumour elsewhere and the uneventful history of previous tumour surgery, metastasis to the orbit is also unlikely with regard to the site and the histology of the lesion.

Thus the most probable tumour origin (though metastatic disease can never be ruled out completely) seems to be (conjunctival?) epithelium which was misplaced during retinal detachment surgery and subsequently underwent malignant transformation. This would also explain the close proximity of the choroidal/orbital tumour to the
Figure 6 Light microscopic appearance of choroidal tumour. Note similarity to orbital tumour (Fig 3) with whorls of prickle cells (arrows) but more necrosis and inflammation. $(S=$ sclera. $R=$ retina, posterior pole to the right. $)(H-E, \times 25$. $)$ Inset shows tumour cells with prickles and adjacent inflammatory cells. $(H-E$, $\times 500$.)

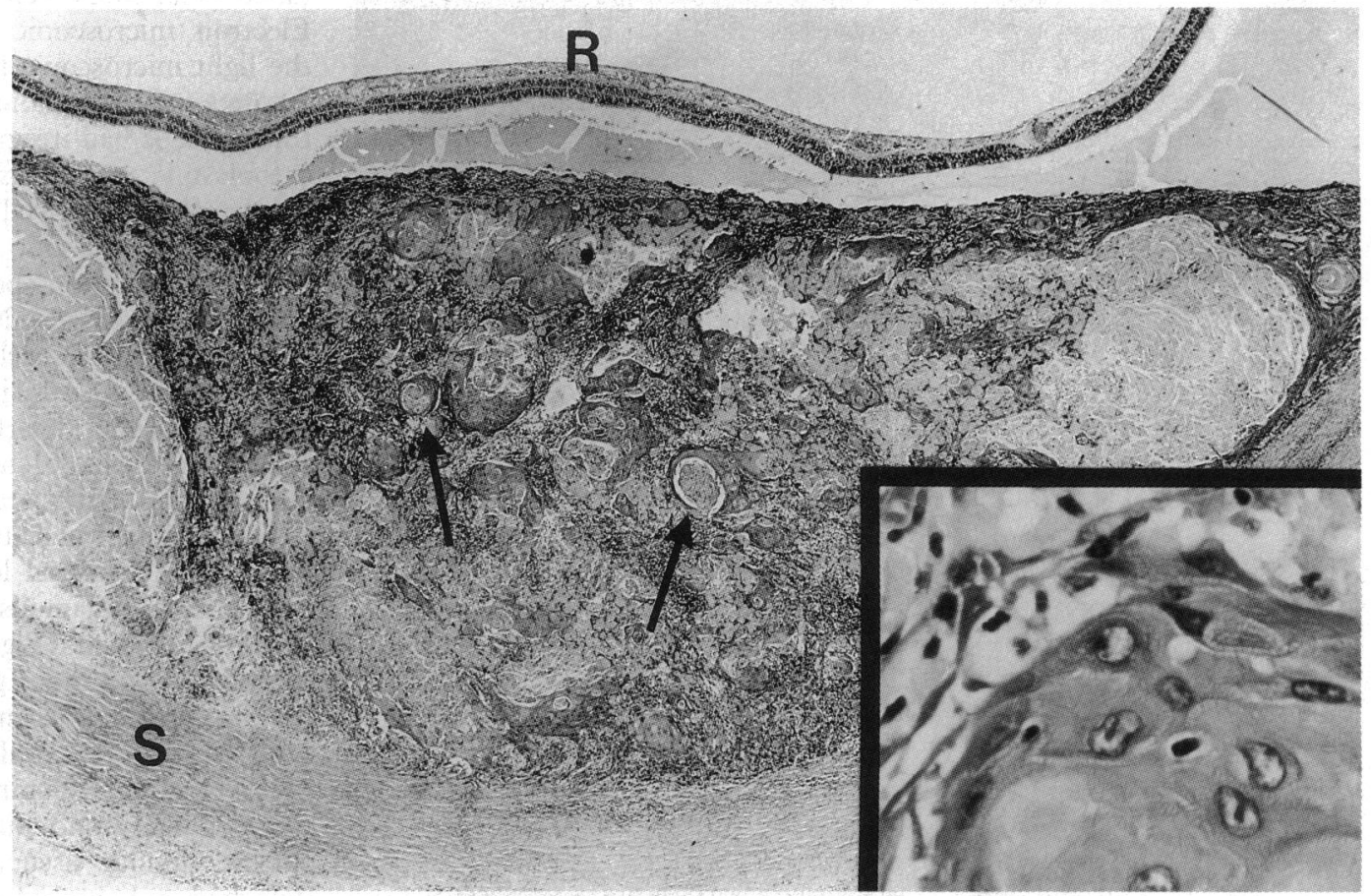

findings in a patient with an orbital and adjacent choroidal squamous cell carcinoma 13 years after an uneventful encircling band procedure. The diagnosis of a well differentiated highly keratinising squamous cell carcinoma seems to be clear. Since there is no primary squamous cell carcinoma of either choroid or orbit, we have three possible mechanisms to explain its presence.

Firstly, invasion of the orbit by a squamous cell carcinoma from the antrum or frontal sinus has to be considered. The clinical history, the site of the tumour, and its cellular characteristics would be consistent with an extension from the maxillary antrum, ${ }^{2}$ but this as well as a lacrimal gland tumour was ruled out by otolaryngological encircling band. The conjunctival strands seen on the section are more likely to be the result of recent surgery - that is, the removal of the orbital tumour - since the cells look healthy and normal despite the wrong environment. They do, however, indicate how easily islands of epithelial cells can be displaced deeply into the adjacent tissue.

We are not aware of any comparable case in the literature. In 1986, at the combined Verhoeff Society and European Ophthalmic Pathology Society meeting in Philadelphia, G J Goder presented a case of an orbital squamous cell carcinoma 53 years after enucleation, but he demonstrated continuity of the tumour with the conjunctival epithelium of the socket. 
Figure 7 Calcification (star) at the site of the encircling band. $(R=$ retina. $S=$ sclera. (Periodic acid Schiff, $\times 50$.)

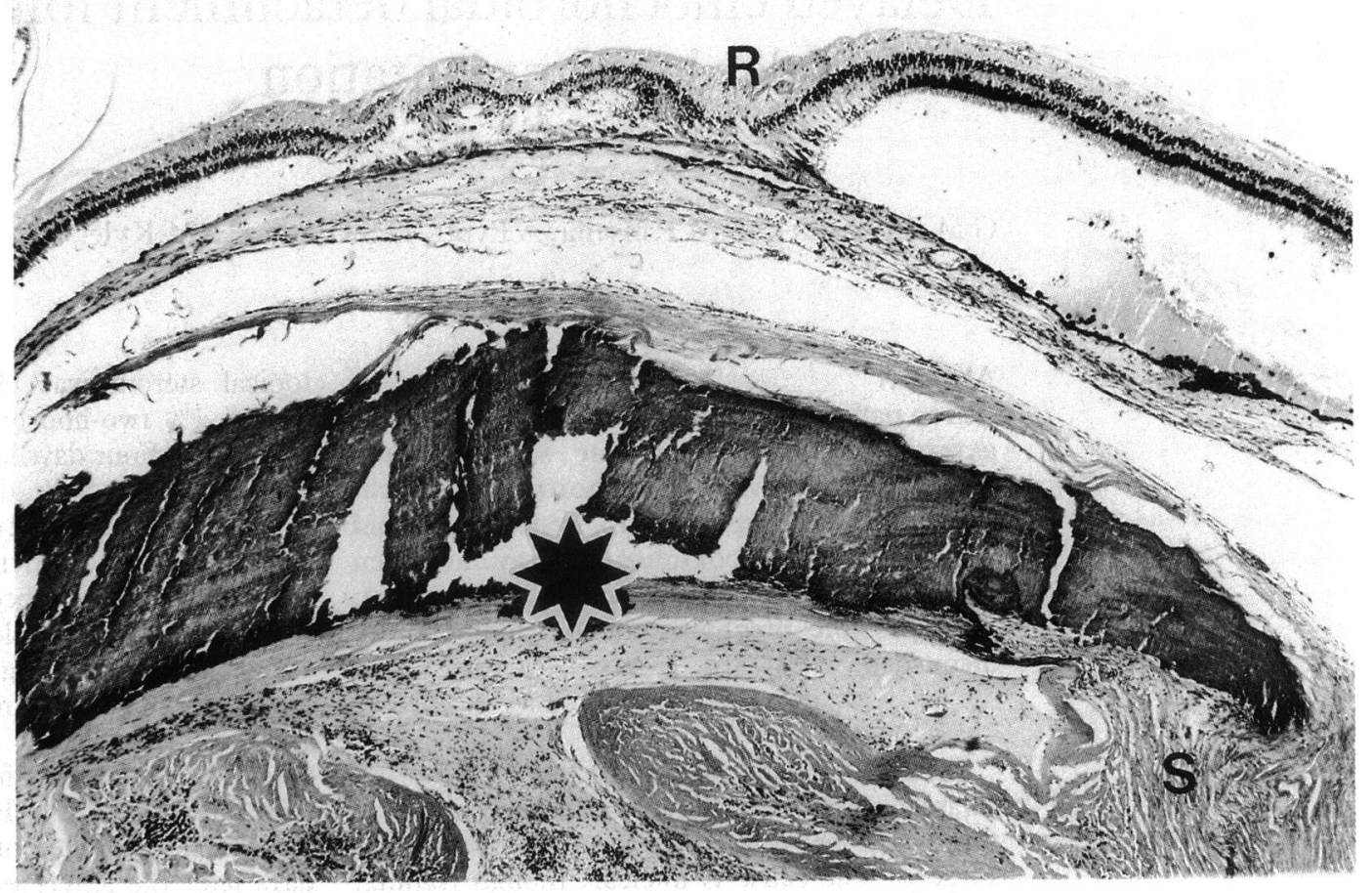

1 Ferry AP, Font RL. Carcinoma metastatic to the eye and orbit. Arch Ophthalmol 1974; 92: 276-86.

2 Jacobiec FA, Font RL. Orbit. In: Spencer W, ed. Ophthalmic pathology: an atlas and textbook. Philadelphia: Saunders, pathology: an atlas

3 Wright JE, Morgan G. Squamous cell carcinoma developing in an orbital cyst. Arch Ophthalmol 1977; 95: 635-7.
4 Jacobiec FA, Font RL. The uveal tract. Tumors. In: Spencer W, ed. Ophthalmic pathology: an atlas

5 Mullaney J. Squamous cell carcinoma of the oesophagus with choroidal metastasis. $\mathrm{Br} \mathcal{F}$ Ophthalmol 1970; 54: 281-3. 